\title{
Design of Exponential Distributed Median Chart with Unknown Parameters
}

\author{
Chengming Cao* and Yizhong Ma \\ School of Economics and Management, Nanjing University of Science and Technology, Nanjing, China, 210094 \\ ${ }^{*}$ Corresponding author
}

\begin{abstract}
It is recommended to monitor the time between events(TBE) when the defect rate is very low. An unbiased median chart is proposed to monitor exponential distributed TBE data and some design parameters are provided for ease of application. For the parameter is usually unknown, the effects of estimation on the chart performance are evaluated in terms of conditional false alarm rate and the conditional average run length(ARL) distribution. It is find that the median statistical can significantly reduce the "practitioner-to-practitioner" variability, and less number of reference samples are needed in phase I control chart. Moreover, the median chart ensures the sensitivity of the process shift to a certain extent. It is shown that the median statistical is an alternative one to monitor the exponential distributed TBE data.
\end{abstract}

Keywords-TBE data; median chart; parameter estimation; conditional run length distribution

\section{INTRODUCTION}

It is recommended to monitor the time between events when the defect rate is very low (per million or per billion) for the conventional attribute control chart for count data are found to be inefficient[1]. The occurrence of the events can be modeled by the homogenous Poisson process, thus the time between consecutive events can be assumed to be independent and identically distributed exponential random variables[2]. It is noticed that the exponential distributed TBE data are highly skewed. The sample median is a robust estimator of location for samples, and it is ease of computation and offers advantages when the population is skewed. Moreover, the med ian chart can maintain the chart's sensitivity in the presence of outliers [3]. Thus, the sample median could be an alternative statistical to monitor TBE data.

Most of the proposed charts have overlooked the parameter estimation, while in practice, the parameters are often unknown and need to be estimated, and control chart performs very differently from the known parameter case, which would affect the practitioners' confidence. Reference [4, 5] highlighted the importance of investigation on the performance of different charts with estimated parameters. Reference[6, 7] focused on the marginal run length distribution and some associated characteristics to evaluate the performance of the control chart. The marg inal run length distribution is averaged over all possible charts constructed using different Phase I samples. Although that the analys is of the marginal run length distribution provides a simple and convenient way to evaluate the performance of the control chart, it can't give the complete picture of the control chart with unknown parameters [7]. The practitioner would most likely have one possible sample of size $\mathrm{N}$ to construct the control chart, and the performance of the control chart will depend on the specific sample. Thus, given different Phase I samples, the control limits and the performance of the corresponding control chart would be very differently for practitioners, which is called the "practitioner-to-practitioner" variability. The conditional run length distribution can give specific information about how poorly or well the control chart performs [8-10].

In this article, we will investigate the median chart by conditional distribution and some related characteristics, such as the conditional false alarm rate (CFAR), the mean and standard deviation of the ARL distribution (AARL and SDARL). The rest of this paper is organized as follows. The median chart and its unbiased design is proposed with both known and estimated parameter case in Section 2. In Section 3 , we evaluate the in-control performance of the median chart by the CFAR, the AARLo and the SDARLo, and the out-of-control performance by the AARL. Finally, conclusions and discussions are drawn in section 4.

\section{DESIGN OF THE MEDIAN CHART WITH BOTH KNOWN AND UNKNOWN PARAMETER CASE}

\section{The known parameter case}

Assume that the occurrence of events follows a homogeneous Poiss on process with constant rate, then the times between consecutive events are exponential distributed random variables. Denote $X$ be the time between two consecutive events, then the probability density function of $X$ is given by:

$$
f(x)= \begin{cases}\lambda \times e^{-\lambda x} & (\mathrm{x}>0) \\ 0 & (\mathrm{x} \leq 0)\end{cases}
$$

where the parameter $\lambda$ is the occurrence rate of the events.

Let $X_{i, 1}, X_{i, 2}, \ldots X_{i, n}, i=1,2, \ldots, N$ be the $i_{\text {th }}$ subgroup, which consists of $\mathrm{n}$ individual observations, Let $Y_{i}$ be the sample median of the subgroup, 


$$
\stackrel{P}{Y}_{i}= \begin{cases}X_{i,((n+1) / 2)} & \text { if } \mathrm{n} \text { is odd } \\ \frac{X_{i,(n / 2)}+X_{i,(n / 2+1)}}{2} & \text { if } \mathrm{n} \text { is even }\end{cases}
$$

Where $X_{i,(1)}, X_{i,(2)}, \ldots X_{i,(n)}, i=1,2, \ldots, N$ is the $i_{\text {th }}$ ordered subgroup. To make the sample median easier and faster to compute, it is usual to assume that the sample size $n$ is an odd value. In the rest of this paper, we will only focus on the case where $n$ is an odd value. It can be easily obtained that the cumulative distribution function of $\hat{Y}_{i}$ is:

$$
F_{Y_{i}}(y)=\left\{\begin{array}{lr}
F_{\beta}\left(1-e^{-\lambda y} \mid \frac{n+1}{2}, \frac{n+1}{2}\right) & (y>0) \\
0 & (y \leq 0)
\end{array}\right.
$$

Where $F_{\beta}\left(\bullet \mid \frac{n+1}{2}, \frac{n+1}{2}\right)$ is the cumulative distribution function of the beta distribution with parameters $\left(\frac{n+1}{2}, \frac{n+1}{2}\right)$.

Denote $U$ and $L$ be the lower and upper control limits, and give a specified false alarm rate $\alpha$ for the median chart with known parameters,

$$
P\left(L \leq \stackrel{P}{i}_{i} \leq U \mid \lambda=\lambda_{0}\right)=1-\alpha
$$

When the process shifts, $\lambda_{1}=\rho \lambda_{0}$, the probability that a sample point falls between the control limits is obtained as follows:

$$
\begin{aligned}
\beta(\rho) & =P\left(L \leq \stackrel{\circ}{i}_{i} \leq U \mid \lambda=\lambda_{1}\right) \\
& =F_{\beta}\left(1-e^{-\lambda_{1} U} \mid \frac{n+1}{2}, \frac{n+1}{2}\right)-F_{\beta}\left(1-e^{-\lambda_{1} L} \mid \frac{n+1}{2}, \frac{n+1}{2}\right) \\
& =F_{\beta}\left(1-e^{-\rho \lambda_{0} U} \mid \frac{n+1}{2}, \frac{n+1}{2}\right)-F_{\beta}\left(1-e^{-\rho \lambda_{0} L} \mid \frac{n+1}{2}, \frac{n+1}{2}\right)
\end{aligned}
$$

The corresponding ARL is,

$$
A R L(\rho)=\frac{1}{1-\beta(\rho)}
$$

For the two-sided control chart, the ARL curve may not approach its maximum value at the in-control state $(\rho=1)$ if the control limits are based on the conventional equal-tail probability limits. Suppose $A R L(\rho)$ is differentiable, to make the ARL approach its maximu $m$ value when $\rho=1$, we make

$$
A R L^{\prime}(1)=0
$$

Thus, for a given false alarm rate $\alpha$, the control limits ( $L$ $U$ ) of the ARL-unbiased median chart can be obtained by the following equations:

$$
\left\{\begin{array}{c}
P\left(L \leq \stackrel{\circ}{Y}_{i} \leq U \mid \lambda=\lambda_{0}\right)=1-\alpha \\
A R L^{\prime}(1)=0
\end{array}\right.
$$

In order to solve (8), let $P\left(\hat{Y}_{i} \leq L \mid \lambda=\lambda_{0}\right)=p_{1}$ and $P\left(\stackrel{\circ}{Y}_{i} \leq U \mid \lambda=\lambda_{0}\right)=1-\alpha+p_{1}$. Then,

$$
\left\{\begin{array}{c}
L=L_{0} / \lambda_{0} \\
U=U_{0} / \lambda_{0}
\end{array}\right.
$$

Where, $\left\{\begin{array}{c}L_{0}=-\ln \left[1-F_{\beta}^{-1}\left(p_{1} \mid \frac{n+1}{2}, \frac{n+1}{2}\right)\right] \\ U_{0}=-\ln \left[1-F_{\beta}^{-1}\left(1-\alpha+p_{1} \mid \frac{n+1}{2}, \frac{n+1}{2}\right)\right]\end{array}\right.$. Substitute (9) into (8), then, the Equation (8) can be solved by the bisection method easily. For ease of application, values of $p_{1}, L_{0}$ and $U_{0}$ of the ARL-unbiased median chart with $\mathrm{n}=3$, 5 and different values of $\alpha$ are listed in Table I.

\section{The estimated parameter case}

We usually estimate the parameters by phase I samples to set up the phase II control limits in the estimated parameter case. Assume that we have a phase I sample of $\mathrm{N}$ failure times each following an exponential distribution with an unknown mean $\lambda_{0}$, the maximum likelihood estimator of $\lambda_{0}$ is:

$$
\$ \frac{N}{\sum_{i=1}^{N} X_{i}}
$$

From the unbiased design of the median chart, the phase II control limits with unknown parameters are given by:

$$
\left\{\begin{array}{l}
L=L_{0} / \$ \\
U=U_{0} / \$
\end{array}\right.
$$

It is obvious that the control limits are random variable for which are the function of the phase I estimator $\$$. Thus, the performance of the median chart with unknown parameters is far different from the known parameter case for the variation of $\$$. The more detailed study will be shown in section 3 .

\section{THE PERFORMANCE EVALUATION OF THE MEDIAN CHART}

Reference[11] investigated the $t_{r}$-chart with unknown parameters. They proposed estimating the parameter from a phase I sample and studied the effects of estimation on the design and performance of the chart by the conditional run length distribution. It is shown that the required phase I sample size needs to be impractically large to make the $t_{r^{-}}$ chart perform as might be expected under the known parameter case because of the "practitioner-to-practitioner" 
variability. In this section, we investigate the performance of the median chart and compare it with the $t_{r}$-chart.

TABLE I. $\mathrm{p}_{1}, \mathrm{~L}_{0}$ AND $\mathrm{U}_{0}$ OF THE ARL-UNBIASED CHART S WITH $\mathrm{n}=3,5$ AND DIFFERENT $\alpha_{0}$

\begin{tabular}{|c|c|c|c|c|c|c|}
\hline \multicolumn{2}{|c|}{$\boldsymbol{\alpha}_{0}$} & $\mathbf{0 . 0 0 1}$ & $\mathbf{0 . 0 0 2}$ & $\mathbf{0 . 0 0 2 7}$ & $\mathbf{0 . 0 0 5}$ & $\mathbf{0 . 0 1}$ \\
\hline \multirow{3}{*}{$\mathrm{n}=3$} & $\mathrm{p}_{1}$ & 0.00083 & 0.00164 & 0.00220 & 0.00402 & 0.00790 \\
\cline { 2 - 7 } & $\mathrm{L}_{0}$ & 0.01689 & 0.02386 & 0.02773 & 0.03778 & 0.05364 \\
\cline { 2 - 7 } & $\mathrm{U}_{0}$ & 4.89232 & 4.51319 & 4.34830 & 4.00816 & 3.62255 \\
\hline \multirow{3}{*}{$\mathrm{n}=5$} & $\mathrm{p}_{1}$ & 0.00079 & 0.00155 & 0.00208 & 0.00379 & 0.00745 \\
\cline { 2 - 7 } & $\mathrm{L}_{0}$ & 0.04480 & 0.05684 & 0.06307 & 0.07823 & 0.10006 \\
\cline { 2 - 7 } & $\mathrm{U}_{0}$ & 3.57051 & 3.31838 & 3.20859 & 2.98178 & 2.72394 \\
\hline
\end{tabular}

Denote $\rho=\lambda_{1} / \lambda_{0}$ be a possible process shift in the phases II.

If $\rho=1$, the process is in control; whereas $\rho<1$ (or $\rho>1$ ), the process is improved (or deteriorated). Let the error factor of the estimator be $\delta=\lambda_{0} / \$$. When the parameter is estimated exactly, $\delta=1$; while $\delta<1$ (or $\delta>1$ ), the parameter is overestimated (or underestimated). The probability of a signal for the median chart with the estimator $\$$ is given by:

$$
\begin{aligned}
& \alpha(\rho, \delta)=P\left(\underline{Y_{i}} \underline{\underline{e}} L \text { or } \underline{Y_{i}^{\prime}} \underline{\underline{\varepsilon}} U \mid \$\right) \\
& =1+F_{\beta}\left(1-e^{-\frac{\lambda_{1}}{\lambda_{0}} \frac{\lambda}{0}^{\infty} L_{0}} \mid \frac{n+1}{2}, \frac{n+1}{2}\right)-F_{\beta}\left(1-e^{-\frac{\lambda_{1}}{\lambda_{0}} \frac{\lambda_{0}}{\$^{*}} U_{0}} \mid \frac{n+1}{2}, \frac{n+1}{2}\right) \\
& =1+F_{\beta}\left(1-e^{-\rho \times \delta \times L_{0}} \mid \frac{n+1}{2}, \frac{n+1}{2}\right)-F_{\beta}\left(1-e^{-\rho \times \delta \times U_{0}} \mid \frac{n+1}{2}, \frac{n+1}{2}\right)
\end{aligned}
$$

Obviously, the probability of a signal is a random variable and the function of $\rho$ and $\delta$, which is the consequence of the parameter estimation. In the estimated parameter case, the estimator $\$$ would be different for different phase I samples, and the probability of a signal would be different for different estimator $\$$. So, the probability distribution of a signal can be calculated conditionally on $\delta$, which reveals the "practitioner-topractitioner" variability. Our main task is to studying the effect of parameter estimation on the chart performance by the conditional ARL distribution.

\section{A Conditional False Alarm Rate of the Median Chart}

The FAR is very essential in designing the control chart, which is the probability of a signal when the process is in control, in other words $\rho=1$. Thus, the CFAR can be expressed by a function of the random variable $\delta$ :

$$
\alpha(\delta)=1+F_{\beta}\left(1-e^{-\delta \times L_{0}} \mid \frac{n+1}{2}, \frac{n+1}{2}\right)-F_{\beta}\left(1-e^{-\delta \times U_{0}} \mid \frac{n+1}{2}, \frac{n+1}{2}\right)
$$

To study the CFAR in a more convenient way, we rewrite $\alpha(\delta)$ as:

$$
\alpha(Y)=1+F_{\beta}\left(1-e^{-\frac{Y}{2(N-1)} \times L_{0}} \mid \frac{n+1}{2}, \frac{n+1}{2}\right)-F_{\beta}\left(1-e^{-\frac{Y}{2(N-1)} \times U_{0}} \mid \frac{n+1}{2}, \frac{n+1}{2}\right)
$$

Where $Y=2(N-1) \times \delta$ follows a chi-square distribution with $2 \mathrm{~N}$ degree of freedom(DOF)[11]. Hence, the $\alpha(Y)$ will have a different value for each $Y$ (or equivalently of the estimator $\$$ ) depending on the different phase I samples. To analyze how the $\alpha(Y)$ value vary depending on the choice of $\mathrm{n}$ and the estimator $\$$, we calculate the $\alpha(Y)$ from Equation(14) for $n=3$, 5 , and $\alpha_{0}=0.002$ at different percentiles of $Y$, for different value of $\mathrm{N}$ and show it in Figure I. At the same time, to compare the median chart with the $t_{r}$-chart easily, the $\alpha(Y)$ of $t_{r}$-chart for $\mathrm{r}=3,5$, at different percentiles of $Y$, for different value of $\mathrm{N}$ are shown in figure I too. Each panel in Figure 1 is divided into two parts with a vertical line drawn through the horizontal axis at the split point. The $\alpha(Y)$ equals to $\alpha_{0}$ at the split point where $Y$ equals to $2(N-1)$. In the left of the split point, the value of $Y$ makes $\delta=Y / 2(N-1)<1$ corresponds to the case of overestimation, and on the contrary, it is the case of underestimation.

Some meaningful observations can be obtained from Figure 1. First, for all choice of $\mathrm{r}$ and $\mathrm{n}$, the value of $\alpha(Y)$ are quite different from the nominal $\alpha_{0}$ in both tail of the distribution of $Y$, and it is more evidently in the lower tail than the upper tail. Second, the $\alpha(Y)$ value are much closer to the nominal $\alpha_{0}$ as $\mathrm{N}$ increases for both the median chart and the $t_{r}$-chart. Third, on every panel in Figure 1, the $\alpha(Y)$ value of the median chart is closer to nominal $\alpha_{0}$ than the $t_{r}$-chart, when $\mathrm{n}=3, \mathrm{r}=3$ (or $\mathrm{n}=5, \mathrm{r}=5$ ). Fourth, when $\mathrm{n}=3$, the $\alpha(Y)$ value of the median chart is closer to the nominal $\alpha_{0}$ than the median chart when $\mathrm{n}=5$ in both the case of overestimation and underestimation.

\section{B Mean and Standard Deviation of the in-control Conditional ARL}

The ICARL is the reciprocal of $\alpha(Y)$, and it can be seen as the function of $Y$ :

$$
\operatorname{ICARL}(Y)=\frac{1}{\alpha(Y)}
$$

The AARLo and SDARLo of the median chart are given by

$$
\begin{aligned}
& A A R L_{0}=E(I C A R L)=\int_{0}^{\infty} \operatorname{ICARL}(y) \varphi(y) d y \\
& S D A R L_{0}=\sqrt{E\left(I C A R L^{2}\right)-[E(I C A R L)]^{2}}
\end{aligned}
$$

Where $E\left(I C A R L^{2}\right)=\int_{0}^{\infty} \operatorname{ICARL}^{2}(y) \varphi(y) d y$, and $\varphi(y)$ is the 
probability density function of a chi-square distribution with $2 \mathrm{~N}$ DOF. The value of AARL 0 and SDARL 0 of the median chart and the $t_{r}$-chart are calculated using Equation(16) and are shown in Table II, for $n=3,5(r=3,5)$ and $N=30,50,100$, 200,500 , or 1000 , for a nominal $\alpha_{0}=0.002$.

It can be seen that the value of AARLo is much closer to the nominal value 500, and the SDARLo value is getting smaller and smaller as $\mathrm{N}$ increase for all charts. Moreover, the AARLo value of the median chart is larger than the $t_{r}$ chart, while the SDARLo value of the median chart is much smaller than the $t_{r}$-chart when $\mathrm{n}=3, \mathrm{r}=3$ (or $\mathrm{n}=5, \mathrm{r}=5$ ).
Reference[12] recommended that the SDARLo value should be within $10 \%$ of the desired ICARL value. It needs nearly 1000 phase I samples to satisfy this for the $t_{r}$-chart when $\mathrm{r}=3$, while it only needs 100 phase I samples for the median chart when $n=3$. The AARLo value when $n=3$ is bigger than the chart's when $n=5$, and the SDARLo value when $n=3$ is smaller than the chart's when $n=5$ for the median chart.

Above all, the median statistical is a more robust monitoring statistical of the chart to monitor the exponential distributed TBE data, and guarantee the nominal in control performance, especially when $n=3$. What's more, comparing

TABLE II. THE AARL AND SDARL $_{0}$ VALUES FOR DIFFERENT VALUES OF N AND n ( $\mathrm{r}$ )

\begin{tabular}{|c|c|c|c|c|c|c|c|c|}
\hline \multirow{3}{*}{$\mathbf{N}$} & \multicolumn{2}{|c|}{$\mathbf{t}_{\mathbf{r}}$-chart } & \multicolumn{2}{|c|}{ median chart } & \multicolumn{2}{|c|}{$\mathbf{t}_{\mathbf{r}}$-chart } & \multicolumn{2}{|c|}{ median chart } \\
\hline & \multicolumn{2}{|c|}{$r=3$} & \multicolumn{2}{|c|}{$\mathbf{n}=\mathbf{3}$} & \multicolumn{2}{|c|}{$r=5$} & \multicolumn{2}{|c|}{$n=5$} \\
\hline & $A A R L_{0}$ & $S D A R L_{0}$ & $A A R L_{0}$ & $S D A R L_{0}$ & $A A R L_{0}$ & $S D A R L_{0}$ & $A A R L_{0}$ & $S D A R L_{0}$ \\
\hline 30 & 414.54 & 163.10 & 415.00 & 91.18 & 363.70 & 160.91 & 386.50 & 109.11 \\
\hline 50 & 439.66 & 147.18 & 440.82 & 69.55 & 398.40 & 145.91 & 418.11 & 87.33 \\
\hline 100 & 464.50 & 122.58 & 466.07 & 43.67 & 436.03 & 122.15 & 450.89 & 58.65 \\
\hline 200 & 480.29 & 97.32 & 481.64 & 24.91 & 462.28 & 98.00 & 472.33 & 36.86 \\
\hline 500 & 491.50 & 67.52 & 492.29 & 10.79 & 482.96 & 68.18 & 488.09 & 16.25 \\
\hline 1000 & 495.63 & 49.58 & 496.08 & 5.53 & 491.04 & 50.32 & 493.88 & 8.51 \\
\hline
\end{tabular}

with the $t_{r}$-chart, the median chart needs less phase I samples to estimate the parameter.

\section{Mean of the out-of-control Conditional ARL}

In order to investigate the out of control performance, we calculate the AARL of the median chart and compare it with the $t_{r}$-chart. For a possible process shift $\rho=\lambda_{1} / \lambda_{0}$ in the phases II, the AARL is calculated as follows:

$$
A A R L=E(C A R L)=\int_{0}^{\infty} \frac{1}{\alpha(y, \rho)} \varphi(y) d y
$$

Where $\varphi(y)$ is the probability density function of a chisquare distribution with $2 \mathrm{~N}$ DOF. The Figure II shows the AARL curves of various phases I samples $\mathrm{N}=30,50,200$, 1000 for both the median chart $(\mathrm{n}=3)$ and the $t_{r}$-chart $(\mathrm{r}=3)$.

In all panels, the AARL curves of the median chart reach their maximum at $\rho=1$, which indicates that the AARL is maximal and unbiased, while the AARL of the $t_{r}$-chart is biased. Second, it can be observed that the $t_{r}$-chart presents AARL values much lower than those in the median chart when the process has improved $(\rho<1)$. Third, the AARL values of the $t_{r}$-chart is much greater than those in the median chart for $\rho>1$, which indicates that the $t_{r}$-chart takes longer time to reflect the process deterioration. Therefore, the median chart can be considered more appropriate for the detection of process deterioration, and people usually care more about that; while the $t_{r}$-chart can be considered more appropriate for the detection of process improvement. What's more, the AARL curve becomes steeper and steeper with $\mathrm{N}$ increase in all panels, which means that the chart is more sensitive in reflecting the process shift of both the median chart and the $t_{r}$-chart.

\section{CONCLUSIONS AND DISCUSSIONS}

In this article, a median chart is proposed to monitor exponential distributed TBE data. The unbiased control limits of median chart are designed, and some design parameters are proposed for ease of application. The performance of the median chart for both the phase I and the 

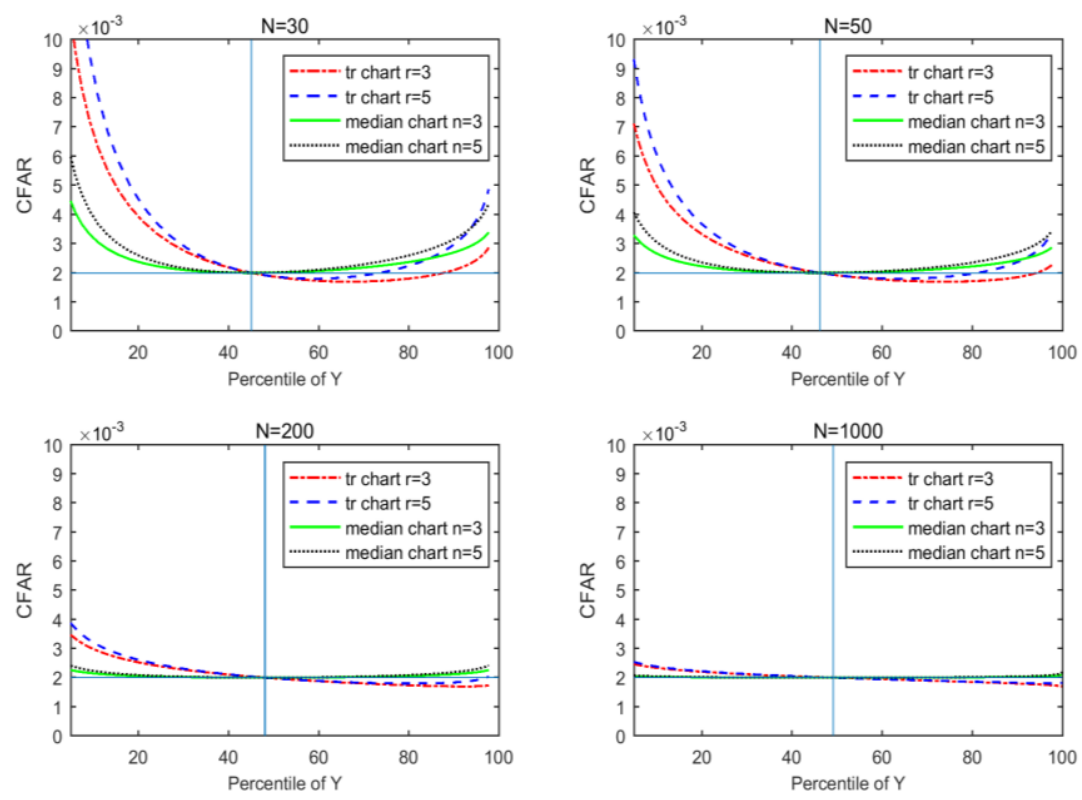

FIGURE I. CFAR OF THE PERCENTILES OF Y FOR N EQUAL TO 30, 50, 200, AND 1000
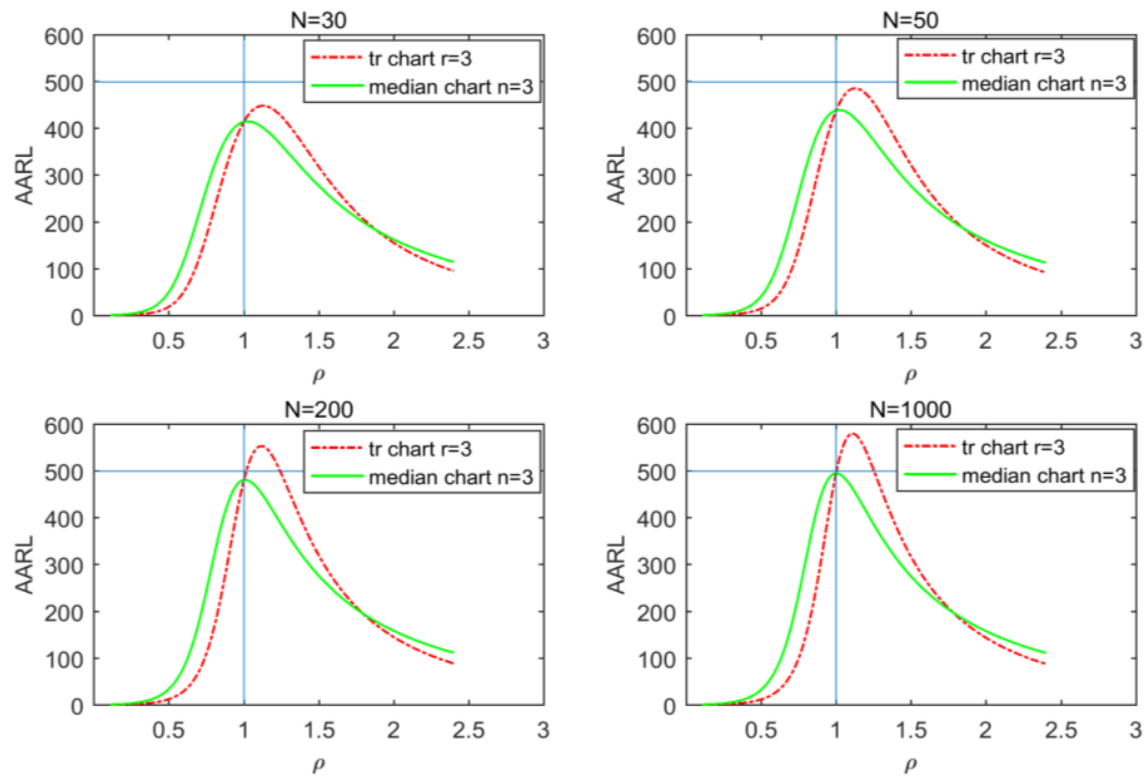

FIGURE II. THE AARL CURVES OF VARIOUS PHASES I SAMPLES FOR N=30, 50, 200, 1000

phase II are investigated by conditional false alarm rate and the conditional ARL distribution. For the phase I, we firstly evaluate the median chart by the CFAR. It is shown that the $\alpha(Y)$ value of the median chart is closer to nominal $\alpha_{0}$ than the $t_{r}$-chart especially when the number of phase I samples is small. What's more, the $\alpha(Y)$ value are much closer to the nominal $\alpha_{0}$ as $\mathrm{N}$ increases for both the median chart and the $t_{r}$-chart. Then, the AARL 0 and SDARLo values of the in-control chart were calculated, which shows that the proposed chart needs less number of phase I samples, in the range of 100 when $n=3$, to assure the in-control performance, which gives confidence to the practitioners to start process monitoring as soon as possible in phase I. The CFAR curve, the AARLo and the SDARLo reveal that the median chart can significantly reduce the "practitioner-to-practitioner" variability compared with the $t_{r}$-chart. For the phase II chart, the AARL curve shows that the proposed chart is more sensitive than the compared chart when the process deteriorates that is people usually care more about; while it needs large nu mber of phase I samples, 
in the range of 1000 , to make sure that the out-of-control performance close to the known parameter case.

The median statistical is a more robust monitoring statistical to monitor the exponential distributed TBE data, and not only guarantee the nominal in control performance but also ensure the sensitivity of the process shift to a certain extent. In addition, the median statistical is much more robust when $n=3$ than the case when $n=5$. We recommend the median chart $(n=3)$ to monitor the exponential distributed TBE data. The drawback is that the median chart is not very sensitive to the process improvement, which needs further study.

\section{REFERENCES}

[1]. D. C. Montgomery, Introduction to statistical quality control. 7th ed. Hoboken, NJ: Wiley 2013.

[2]. F. F. Gan, "Designs of one- and two-sided exponential EWMA charts." Journal of Quality Technology, vol 30: pp. 55-69. 1998.

[3]. M. B. C. Khoo, "A Control Chart Based on Sample Median for the Detection of a Permanent Shift in the Process Mean." Quality Engineering, vol 17(2): pp. 243-257. 2005.

[4]. S. Psarakis, A. K. Vyniou, and P. Castagliola, "Some Recent Developments on the Effects of Parameter Estimation on Control Charts." Quality and Reliability Engineering International, vol 30(8): pp. 1113-1129. 2014.

[5]. W. A. Jensen, L. A. Jones-Farmer, C. W. Champ, and W. H. Woodall, "Effects of parameter estimation on control chart properties: A literat ure review." Journal of Quality Technology, vol 38(4): pp. 349364. 2006.

[6]. S. Chakraborti, "Run length, average run length and false alarm rate of Shewhart X-bar chart: Exact derivations by conditioning." Communications in Statistics-Simulation and Computation, vol 29(1): pp. 61-81.2000.

[7]. G. Ozsan, M. C. Testik, and C. H. Weiss, "Properties of the Exponential EWMA Chart with Parameter Estimation." Quality And Reliability Engineering International, vol 26(6): pp. 555-569. 2010.

[8]. C. S., "Parameter estimation and design considerations in prospective application of X-bar chart." Journal of Applied Statistics, vol 3: pp. 439-459.2006.

[9]. E. EK., L. LD., and C. S., "Effect of the number and size of the phase I sample on the phase II performance of S2 and S control charts." Journal of Quality Technology. 2014.

[10].N. A. Saleh, M. A. Mahmoud, M. J. Keefe, and W. H. Woodall, "The Difficulty in Designing Shewhart (X)over-bar and X Control Charts with Estimated Parameters." Journal of Quality Technology, vol 47(2): pp. 127-138.2015.

[11].N. Kumar and S. Chakraborti, "Phase II Shewhart-type Control Charts for Monitoring Times Between Events and Effects of Parameter Estimation." Quality and Reliability Engineering International, vol 32(1): pp. 315-328. 2016.

[12].M. Zhang, F. M. Megahed, and W. H. Woodall, "Exponential CUSUM Charts with Estimated Control Limits." Quality and Reliability Engineering International, vol 30(2): pp. 275-286. 2014. 